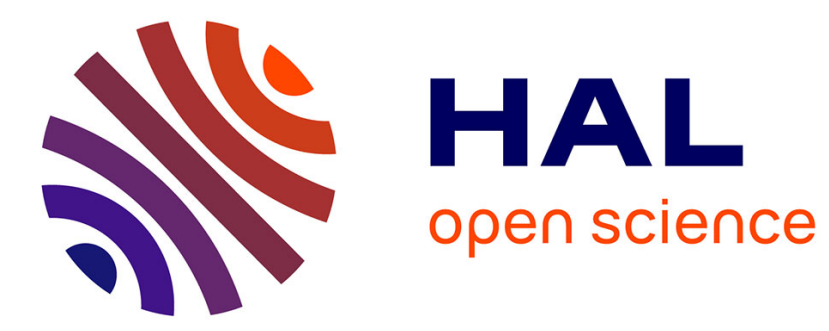

\title{
Afrique du Sud : à la recherche de la ville perdue Philippe Gervais-Lambony
}

\section{To cite this version:}

Philippe Gervais-Lambony. Afrique du Sud : à la recherche de la ville perdue. Multitudes, 2004, 17 (3), pp.157. 10.3917/mult.017.0157 . hal-02320050

\section{HAL Id: hal-02320050 \\ https://hal.parisnanterre.fr/hal-02320050}

Submitted on 18 Oct 2019

HAL is a multi-disciplinary open access archive for the deposit and dissemination of scientific research documents, whether they are published or not. The documents may come from teaching and research institutions in France or abroad, or from public or private research centers.
L'archive ouverte pluridisciplinaire HAL, est destinée au dépôt et à la diffusion de documents scientifiques de niveau recherche, publiés ou non, émanant des établissements d'enseignement et de recherche français ou étrangers, des laboratoires publics ou privés. 
Afirique

du Sud:

à la recherche

de la

ville perdue

Philippe

Gervais-Lambony 
Il y a, tout près du centre-ville de Boksburg, un petit lac. Nous sommes dans l'agglomération du Witwatersrand, cette région urbaine des hautes terres intérieures sud-africaines née à la fin du XIX ${ }^{\mathrm{e}}$ siècle à partir de l'exploitation de gisements aurifères. Au centre de cette région se trouve la ville de Johannesburg, de part et d'autre s'étendent des villes moyennes (environ 200000 habitants chacune ${ }^{\mathrm{I}}$ ) jointives, formant vers l'ouest le West Rand, vers l'est l'East Rand. C'est à ce deuxième ensemble qu'appartient Boksburg.

Les abords du lac de Boksburg sont désuets, aux deux sens du mot: d'allure surannée, il sont aussi aujourd'hui un espace «tombé en désuétude». Point de promeneurs sur les berges parsemées d'aires de piquenique, les jets d'eau ne sont jamais ouverts, l'embarcadère des pédalos est à l'abandon et les guirlandes d'ampoules multicolores ne sont allumées qu'une fois l'an, pendant les fêtes de Noël. Mais l'endroit est bien entretenu et la forte grille de couleur vert sombre qui entoure le lac est régulièrement repeinte.

Au sud du lac, les quelques rues commerçantes du centre de Boksburg, bordées de petits immeubles, sont animées dans la journée, les piétons sont surtout des citadins noirs qui travaillent au centre, y font escale dans leur trajet quotidien ou viennent acheter des produits dans les boutiques bon marché. Le soir, le centre est désert, à l'exception des prostituées qui y prennent position, surtout aux abords du Central Hotel, vieux bâtiment de style victorien qui a connu des jours meilleurs.

Au nord du lac se dresse le haut terril d'une mine d'or, fermée au début des années I 960. Cette masse grisâtre est un des vestiges de l'activité qui a donné le jour à Boksburg et a modelé cette petite société urbaine. La compagnie minière East Rand Proprietary Mines (ERPM) était le premier employeur local et le premier investisseur immobilier de la région. De la mine sont nées aussi les industries métallurgiques et chimiques, contrôlées par les mêmes capitaux.

\section{la ville autour du lac}

Commencer la description de Boksburg par celle de son lac n'est pas un effet de style. Créé en même temps qu'étaient creusés les puits des mines d'or, le lac de Boksburg devait servir d'espace récréatif pour la petite communauté blanche de la ville, typiquement sud-africaine. Très tôt, dès avant la mise en place de l'apartheid à partir de $1948^{2}$, fut exclue de la fréquentation de ce lieu la population "non-blanche " de la ville, à l'exception des "nannies" qui poussaient les landaus d'enfants, des jardinierset des serveurs lors des garden-parties organisées par l'ERPM.

Cette société de ville minière s'accommoda fort bien de la mise en 
place de la législation de l'apartheid qui figeait la ségrégation raciale et sociale. Spatialement, la conséquence de l'apartheid à Boksburg fut le déplacement des populations noires, durant les années I960, vers un nouveau "quartier ", Vooslorus, ensemble de maisons individuelles locat ives construites par l'État, c'est-à-dire un township situé à une dizaine de kilomètres au sud du centre-ville. Le quartier où habitaient ensemble les "non-blancs " 3 , Reiger Park, à deux kilomètres du centre-ville, fut déclaré réservé aux colorés. Un peu plus tard fut construit, entre Vosloorus et Reiger Park, le quartier de Villa Lisa, réservé aux personnes d'origine indienne. Pendant ce temps, de part et d'autre du centre, se développaient les banlieues résidentielles "blanches", dans des limites municipales qui n'englobaient pas Vosloorus, géré directement par l'administration centrale des "Affaires Africaines". Cette rationalis ation de la ségrégation légale mettait à distance les résidences des non-Blancs, libérait la municipalité blanche de la gestion de la main-d'œuvre noire, de son logement et de son éducat ion. Mais en même temps cette ségrégation maintenait cette main-d'œuvre nécessaire à proximité et, par l'interdiction des activités commerciales dans les townships, livrait aux commerçants blancs du centre-ville une clientèle captive et nombreuse.

À partir de la fin des années I 970, ce système s'effrita. La révolte des townships, commencée à Soweto en 1976, s'inscrivait dans un contexte plus large de crise du système urbain. À Boksburg, d e venue ville industrielle à partir des années 1960 , comme ailleurs, le contrôle des quartiers africains devint de plus en plus difficile. La situation de révolte rampante ou affichée dans les townships, les boycotts, les grèves, concordaient avec la baisse rapide des emplois industriels (et la disparition des emplois miniers), c'est-à-dire le déclin de l'économie dite fordiste qui altéra la relation entre la ville blanche et la ville noire. La création en I982 des Black Local Authorities (BLA), municipalités noires élues mais soumises au contrôle des autorités blanches et dépourvues de moyens financiers propres, ne résolut rien : rejetées, boycottées, les BLA ne fonctionnèrent que bri èvement. Le véritable pouvoir local dans les townships et dans les camps informels qui se développaient revint de fait au mouvement des associations citadines, les civics. Ceux-ci, proches de l'ANC interdit, quadrillaient l'espace de la ville noire, du comité de rue à la structure régi onale puis nationale. Les civics mobilisaient les citadins dans un combat commun pour une autre ville, ou une autre vie en ville; ils faisaient ainsi la ville, c'est-à-dire qu'ils constituaient les habitants en communauté, ce que ne parvenaient plus du tout à faire les autorités légales.

Un événement particulier permet de mieux comprendre fonctionnement et objectifs des civics et de mettre en relief leur rôle essentiel dans 
la constitution d'une identité urbaine dans les années 1980. En I988, les élections municipales à Boksburg furent remportées par le Parti Conservateur (CP). Extrémistes de l'apartheid, les dirigeants de parti refusaient les réformes des années I980. Leur premier acte à l'échelle municipale fut de remettre en vigueur l'ensemble des règles plus ou moins abandonnées de l'apartheid dit "mesquin " : bancs séparés, entrées distinctes dans les commerces et lieux publics pour Blancs et non-Blancs. Au centre-ville, un règlement municipal fut édicté, interdisant la fréquentation du bord du lac aux non-Blancs. Dans une période d'apartheid finissant, ces mesures provoquèrent la colère des habitants des quartiers non-blancs. C'est le mouvement des civics qui organisa la réponse collective, laquelle transcenda les séparations « raciales » que l'apartheid avait créées : les habitants des quartiers indien, coloré et noir étaient unis dans la même revendication au droit d'usage du centre-ville et, symboliquement, du lac de Boksburg. Une partie de la population blanche anglophone soutint aussi le mouvement. Les civics organisèrent le boycott des commerces de Boksburg, mettant en place des systèmes de transport en taxis collectifs pour que les habitants de Reiger Park, Villa Lisa et Vosloorus puissent aller faire leurs courses soit à Johannesburg, soit dans les municipalités visines. Le Boksburg Boycott, resté célèbre comme modèle de lutte contre l'apartheid, fut un succès : plusieurs conseillers municipaux démissionnèrent assez rapidement, et la municipalité revint au Parti National qui abolit les règlements du CP. Ceci démontre assez que la ville d'apartheid était un système dans lequel les uns avaient besoin des autres, et que les associations citadines savaient maintenir un lien social de nature politique : la ville d'apartheid «faisait ville», malgré tout.

\section{de la ségrégation à la fragmentation, la ville perdue ?}

Que reste-t-il de nos amours? Les villes sud-africaines ont été au cœur de tous les espoirs de l'après-apartheid. Parti de Soweto en I976, le vaste mouvement de révolte des populations citadines n'a pas été pour rien dans la chute du régime, achevée tout à fait en avril I994 avec l'élection de Nelson Mandela, "my black president " chanté par Brenda Fassie. La fin de l'apartheid fut un rêve, ou plutôt des rêves. Dans le champ urbain, politiciens, aménageurs, intellectuels, activistes, militants, citadins ont rêvé d'une ville à venir, sur les ruines du système ségrégatif et raciste de l'apartheid : une ville équitable, redistributrice, déségréguée. Les concepts de compact city et de one city servaient de fondement à ces rêves de ville parce qu'il s'agissait de prendre le contre-pied de la ville d'apartheid: les concepteurs de cette dernière avaient mis à l'écart les populations non-blanches dans les townships, et laissé se développer se- 
lon les lois du marché les quartiers résidentiels blancs très peu denses. Étalement et éclatement spatial caractérisaient donc la ville d'apartheid autant que la division administrative.

Relativement aux rêves du début des années I990, les villes sud-africaines ont peu changé dans leur forme. On sait que «la forme spatiale de la ville n'est jamais un reflet clair et parfait des processus urbains " 4 : les processus plus anciens ne s'arrêtent pas brusquement pour laisser place à d'autres ; les acteurs, surtout économiques, ne changent pas de strat é gie brutalement; le bâti et les infrastructures, enfin, ont une force d'inertie considérable. Ainsi le présent d'un espace urbanisé est toujours à la fois encore "du passé " (fixité, inertie) et déjà "dans le futur " (processus en cours).

Mais dans le cas sud-africain, il y a autre chose. Beaucoup commencent à penser ou dire que non seulement la forme urbaine ne change pas ou peu, mais aussi que la ville-même est menacée de disparition parce que le lien social citadin s'estompe. C'est une hypothèse crédible : on serait passé d'une ville racialement ségréguée à une ville "fragmentée ». La ville de l'apartheid, pour inégalitaire qu'elle fût, était un système urbain qui rattachait les citadins les uns aux autres. Les habitants des townships étaient maintenus dans une position sociale inférieure et cantonnés dans leurs quartiers, mais ils étaient employés (exploités) par l'économie de la ville, dont ils constituaient la main-d'œuvre. Les habitants de la ville "blanche " étaient les maîtres et les privilégiés, mais cette main-d'œuvre leur était nécessaire. Ces différents groupes se rencontraient : au centreville dans la journée, sur les lieux de travail, ou simplement dans les maisons où travaillaient des femmes et des hommes noirs comme domestiques des Blancs. La ville d'aujourd'hui ne fait peut-être plus système. La main-d'œuvre est largement inutile — près de $40 \%$ de sans-emploi parmi les actifs l'attestent. Le passage du fordisme au post-fordisme, en Afrique du Sud comme ailleurs, semble briser la ville $5: 1$ 'A frique du Sud a perdu plus de 500000 emplois, essentiellement dans l'industrie, depuis I994. Dans le même temps, la peur de l'autre, liée aux perceptions de la criminalité, a fait se fermer quartiers, rues, espaces publics. Mais il y a une autre raison à la fin de la ville en tant qu'entité, plus spécifique peut-être à l'Afrique du Sud : le fondement de la cohésion sociale était, pour la majorité des citadins, la lutte politique, à tout le moins la situation commune d'opprimé (et d'opprimant pour certains). Ce lien social né dans et par le politique s'est-il effacé ?

Revenons à l'image du Boksburg d'aujourd'hui présentée au début de ce texte. Les berges du lac sont désertes : cet espace public revendiqué à la fin des années I 980 semble inutile aujourd'hui. Le centre-ville, 
objet des conflits d'il y a quinze ans, est en perte de vitesse économique face au développement d'un vaste centre commercial au nord de l'exmunicipalité, l'East Rand Mall, la plus grande surface commerciale du Witwtatersrand. Les soirs de fin de semaine, c'est dans ce shopping mall que vont au restaurant ou au cinéma les résidents blancs des quartiers aisés et moyens de Boksburg, qui sortent de leurs villas barricadées ou de leurs complexes résidentiels fermés. Seule une élite africaine nouvelle (et motorisée) peut fréquenter ce centre depuis des quartiers résidentiels où elle s'est récemment installée. Depuis Vosloorus ou Reiger Park, c'est une longue route, et il n'y a pas de ligne directe de taxi. Au total, le centre-ville est aujourd'hui ouvert et africanisé, mais il a perdu la substance même qui en faisait un enjeu : gagner l'accès à cet espace public-là a peut-être été un marché de dups.

De nouveaux clivages sociaux sont ainsi apparus, plus forts que naguère, à l'intérieur de la population noire. Selon certains auteurs sudafricains ${ }^{6}$, ce sont ces fractures-là qui priment dans la ville d'aujourd'hui. A-t-on ainsi perdu la ville en tant que "bien commun" de ses habitants, ou du moins d'une partie d'entre eux, unis du temps du Boksburg Boycott? Outre le seul fait que la ségrégation sociale dans les townships tels que Vosloorus s'accentue peut-être parce que ceux qui en ont les moyens ont tendance à partir s'installer ailleurs, l'émergence de nouveaux groupes sociaux africains se perçoit aussi au quotidien dans des lieux particuliers.

\section{la ville pourtant?}

Bien des aspects de la politique urbaine actuelle ont cependant pour objectif de maintenir ou renforcer la "fabrique» d'une société urbaine. La réforme des autorités locales a dans ses grandes lignes consisté à réunir les anciennes municipalités noires et blanches. Dans le cas des grandes agglomérations, c'est la métropolisation politico-administrative qui a été choisie. On insiste beaucoup sur la démocratie participative, qui est censée s'exprimer au niveau de la circonscription électorale dans le cadre d'un processus de consultation des populations. Des ward committee (comités de quartiers d'une dizaine de membres volontaires) sont ainsi organisés par et autour de l'élu local; ils sont censés animer la vie démocratique du quartier, faire circuler les informations, organiser des réunions... Quand les circonscriptions sont "mixtes ", c'està-dire à cheval sur des quartiers qui relevaient naguère de "groupes raciaux " différents, se produit au moins un contact formel entre "communautés ". Dans les autres cas, le comité a aussi d'autres fonctions : pallier la disparition de l'implication citadine dans la lutte politique, 
tout en "contournant » les civics qui, de leur côté, se considèrent encore souvent comme les meilleurs représentants des citadins.

Un dimanche de 2003, une assemblée de circonscription se tenait à Vosloonus, dans le ward numéro 34. Dans une circonscription qui compte en moyenne II ooo habitants, quelque I50 personnes étaient présentes pour écouter le discours du conseiller et débattre. Après une prière d'ouverture, comme dans toute réunion politique en Afrique du Sud, le conseiller expose son programme: créer de nouveaux espaces de jeux pour les enfants, mais en confier l'entretien aux citadins, améliorer le ramassage des ordures en responsabilisant la " communauté ", améliorer la sécurité routière en imposant les limitations de vitesse, développer l'agriculture intra-urbaine pour créer des emplois, réduire la criminalité, mais là aussi par l'appel à la communauté : "si vous achetez bon marché des produits volés, il y aura toujours des vols! ». Au moment où le maire déclare dans son discours de début d'année que l'objectif est de faire de la métropole un "business paradise", on appelle les citadins à prendre en charge eux-mêmes la gestion de leur quartier. Il y a derrière ce discours participatif toute l'ambiguïté d'une politique urbaine qui vise aujourd'hui à développer certains espaces pour favoriser le développement économique, et à se désengager des zones les plus pauvres. Mais, à la suite du discours du conseiller, les interventions du public se concentrent sur d'autres points, notamment le crime: plusieurspersonnes se lèvent pour dire à quel point la police est incompétente, c ertains soulignent que "c'était mieux avant ", que le problème vient " de la corruption des hommes noirs qui sont au pouvoir ". D'autres racontent leurs mauvaises expériences à la clinique du quartier: "l'aspirine ne suffit pas contre le sida!". Plusieurs autres personnes répondent que l'on ne peut pas dire que les choses allaient mieux sous l'apartheid, où l'on risquait sans cesse d'être arrêté pour un nen... Le débat tourne plusieurs minutes sur cette question. Finalement quelqu'un déclare que ce qui était mieux sous l'apartheid, c'était que les citadins venaient aux meetings politiques, que les gens participaient, alors qu'aujourd'hui ils attendent d'être servis.

Ce type de réunion de quartier constitue bel et bien un exemple de démocratie locale, avec tous ses défauts mais aussi avec l'extraordinaire liberté de parole qui la caractérise. Il est toutefois clair que l'échelle de la circonscription ne favo rise pas l'unité de l'ensemble urbain, mais plutôt une identification forte au quartier. Finalement, la ville, en tant qu'entité politique une, se perd là aussi.

C'est peut-être ailleurs qu'il faut chercher les modes de construction de l'identité citadine, dans un certain rapport au passé et une mémoire commune. L'Afrique du Sud est empreinte de nostalgie : popu- 
lations déplacées, terres volées, noms modifiés, espaces manipulés... C’est le pays de la confusion entre passé et présent, un pays où le passé est sans cesse surgissant, tout en étant sans cesse étouffé. À la sortie du centreville de Brakpan, le long d'une rue appelée Location Road, se trouve un vaste terrain vague: c'est ce qui reste de l'ancienne location, c'est-à-dire le quartier qui abritait la population non-blanche de Brakpan avant que les autorités de l'apartheid en ordonnent la destruction et le déplacement vers les townships. Les rues goudronnées sont toujours là, le sol est parsemé des fondations de maisons détruites et, dans une des rues, une vieille boîte aux lettres tient toujours debout. La location de Brakpan a commencé à être rasée à partir de la fin des années I960, mais elle a résisté longtemps : dix ans plus tard, la destruction n'était pas achevée. Pour beaucoup d'habitants des townships de l'East Rand subsiste la nostalgie des locations ${ }^{7}$. Ils en ont été chassés, adultes ou dans leur enfance, ils s'en souviennent et idéalisent ce souvenir. Ils continuent d'ailleurs souvent de se considérer comme originaires de là... La location était un lieu où chacun avait un "droit à la ville ": on pouvait y être propriétaire, on habitait près du centre, les bars, clandestins, était nombreux. Ces quartiers étaient aussi sur-peuplés, mal équipés, fortement ségrégués, mais le temps des locations a donné naissance au mythe urbain essentiel: celui d'une véritable citadinité africaine perdue. Si ce mythe a résisté à l'apartheid - qui a tenté d'effacer cette ville-là, physiquement et symboliquement —, il est permis d'espérer qu'il résistera aussi à la mondialisation post-moderne, même si les acteurs de celle-ci sont plus habiles à récupérer les mythes pour les vider de leur sens.

(I) L'ensemble du Witwatersrand compte environ six millions d'habitants.

(2) Année de l'arrivée au pouvoir du National Party avec pour slogan, apartheid.

(3) C'est-à-dire les personnes d'ori gine indienne, les Noirs sud-africains ou d'origine étrangère et ceux que l'on désignait en Afrique du Sud comme "Coloured " parce qu'ils n'étaient ni Blancs, ni Noirs, ni Indiens. On est contraint aujourd'hui encore de recourir à ces désignations racistes pour expliquer l'Afrique du Sud. J'utilise le mot français "coloré " pour traduire coloured, plus proche de la réalité de ce groupe très divers que le terme "métis".

(4) Beauregard R. A. et Haila A., "The Unavoidable Continuities of the City ", in Marcuse P. et Van Kempen R., Globalising Cities, A New Spatial Order?, Oxford, Blackwell Publishers, 2000, page 24 .

(5) Sur cette analyse de la fragmentation sociale voir Naez-Bouchanine, La Fragmentation urbaine en question, Paris, l'Harmattan, 2002.

(6) Voir Beall J., Crankshaw O, Parnell S., Uniting a Divided City. Governance and Social Exclusion in Fohannesburg, Londres, Earthscan, 2002.

(7) Toutes les villes sud-africaines avaient leur location. Les plus emblématiques et les plus connues sont restées celle du Cap, District Six, et celle de Johannesburg, Sophiatown. Cette dernière a été rasée et remplacée par un quartier résidentiel blanc baptisé "Triomf ". En I999, le nom de Sophiatown a été rétabli (voir Guillaume P, fohannesburg, Géographies de l'exclusion, Paris, Karthala, 200I, chapitres 2 et 4 ). 